\title{
The Orientational Pair Correlation Functions in a Dense Hard Sphere Fluid at Long Times
}

\author{
Jan A. Leegwater ${ }^{1}$ and Henk van Beijeren ${ }^{1}$
}

Received June 5, 1989; revised June 15, 1989

\begin{abstract}
The two-particle contribution to the potential part of the stress tensor autocorrelation function of a dense hard sphere fluid is studied. It is shown that the long-time decay is given as the solution of a diffusion equation for the relative particle in a potential of mean force. The diffusion constant needed in order to accurately reproduce molecular dynamics results is found to be somewhat lower than the self-diffusion constant.
\end{abstract}

KEY WORDS: Self-diffusion; pair diffusion; potential of mean force.

\section{INTRODUCTION}

In a recent molecular dynamics study of dense hard sphere fluids Ladd et $a l^{(1,2)}$ found that the pair contribution to the potential part of the transverse stress tensor autocorrelation function is described by a stretched exponential, only for very long times they observed a transition to the algebraic $t^{-7 / 2}$ long-time tail that is given by hydrodynamic mode coupling theory. A different time behavior is given by molecular scale mode coupling theories. In recent years a number of mode coupling calculations have appeared for the full stress tensor autocorrelation function ${ }^{(3-5)}$ which yield results in the right order of magnitude. The present work is based on these ideas, but the analysis is carried through much further by including recollisions as well as static correlations. We will show below that a calculation analogous to ref. 4 , as performed by Kirkpatrick, ${ }^{(6)}$ gives only a semiquantitative agreement with simulations. The simulations can, however, success-

\footnotetext{
${ }^{1}$ Institute for Theoretical Physics, University of Utrecht, 3508 TA Utrecht, The Netherlands.
} 
fully be understood in terms of a diffusion model, where the interaction between the particle pair is described by a potential of mean force.

For a system of $N$ hard spheres with diameter $\sigma$ and mass $m$ confined in a box with volume $V$ the potential part of the stress tensor is given by

$$
S_{x y}^{p}=-\frac{1}{V} J_{x y}^{p}
$$

where the potential part of the transverse momentum current $J_{x y}^{p}$ can be written as

$$
J_{x y}^{p}=\sum_{i<j} m\left(\mathbf{v}_{i j} \cdot \hat{\mathbf{r}}_{i j}\right)^{2} \theta\left(-\mathbf{v}_{i j} \cdot \hat{\mathbf{r}}_{i j}\right) \frac{x_{i j} y_{i j}}{\sigma} \delta\left(r_{i j}-\sigma\right)
$$

where $\hat{\mathbf{r}}=\mathbf{r} / r$. The potential part of the stress tensor correlation function $\left\langle J_{x y}^{p}(0) J_{x y}^{p}(t)\right\rangle$ is a four-particle correlation function. It can be decomposed into two-, three-, and four-particle contributions. The two-particle contribution is the simplest one to study, and here we restrict ourselves to this contribution only. Define the normalized two-particle correlation function as

$$
\rho^{(J)}(t)=\frac{\beta t_{e}}{V \eta_{e}}\left\langle\sum_{i<j} J_{x y}^{i j}(0) J_{x y}^{i j}(t)\right\rangle
$$

where $t_{e}$ is the (Enskog) mean free time and $\eta_{e}$ is the Enskog shear viscosity. ${ }^{(7)}$ For long times the velocity dependence of $J_{x y}^{p}$ has been shown to be immaterial, ${ }^{(1)}$ so we may equally well consider the following microscopic densities:

$$
K_{x y}^{i j}=\left(\mathbf{v}_{i j} \cdot \hat{\mathbf{r}}_{i j}\right) \theta\left(-\mathbf{v}_{i j} \cdot \hat{\mathbf{r}}_{i j}\right) \frac{x_{i j} y_{i j}}{\sigma^{2}} \delta\left(r_{i j}-\sigma\right)
$$

and

$$
L_{x y^{\prime}}^{i j}=\frac{x_{i j} y_{i j}}{\sigma^{2}} \delta\left(r_{i j}-\sigma\right)
$$

Keeping in mind that the average over the Maxwell distribution of $\left(\mathbf{v}_{i j} \cdot \hat{\mathbf{r}}_{i j}\right)^{2} \theta\left(-\mathbf{v}_{i j} \cdot \hat{\mathbf{r}}_{i j}\right)$ is $1 / \beta m$, and that the average of $\left(\mathbf{v}_{i j} \cdot \hat{\mathbf{r}}_{i j}\right) \theta\left(-\mathbf{v}_{i j} \cdot \hat{\mathbf{r}}_{i j}\right)$ is $1 /(\pi \beta m)^{1 / 2}$, we define the corresponding two-particle correlation functions

$$
\rho^{(K)}(t)=\frac{m \sigma^{2} \pi t_{e}}{V \eta_{e}}\left\langle\sum_{i<j} K_{x y}^{i j}(0) K_{x y}^{i j}(t)\right\rangle
$$


and

$$
\rho^{(L)}(t)=\frac{\sigma^{2} t_{e}}{V \beta \eta_{e}}\left\langle\sum_{i<j} L_{x y}^{\ddot{j}}(0) L_{x y}^{i j}(t)\right\rangle
$$

If the velocity dependence can indeed be factorized for long times, we have the following relation:

$$
\rho^{(J)}(t) \simeq \rho^{(K)}(t) \simeq \rho^{(L)}(t)
$$

In the molecular dynamics simulations of Ladd et al. ${ }^{(1)}$ this factorization has been found to hold for $\rho^{(J)}$ and $\rho^{(K)}$ at times larger than about ten mean free times.

In the next section we study a generalization of $\rho^{(L)}(t)$,

$$
\rho_{l}(t)=\sigma^{2}(N-1)\left\langle\delta\left(r_{12}-\sigma\right) Y_{i m}^{*}\left(\hat{\mathbf{r}}_{12}\right) \delta\left(r_{12}(t)-\sigma\right) Y_{l m}\left(\hat{\mathbf{r}}_{12}(t)\right)\right\rangle
$$

with $Y_{I m}$ a spherical harmonic. Due to the homogeneity of an equilibrium fluid, (1.9) is independent of $m$. Using that $Y_{2+2}=(15 / 32 \pi)^{1 / 2}(x \pm i y)^{2}$, we find the function $\rho_{2}$ to be related to $\rho^{(L)}$ as

$$
\rho^{(L)}(t)=\frac{2 \pi n t_{e}}{15 \beta \eta_{e}} \rho_{2}(t)
$$

Besides $\rho_{2}, \rho_{0}$ is an interesting quantity as well, as it describes the probability of a recollision for the particles 1 and 2. Furthermore, if the velocity factorization holds, $\rho_{0}$ is proportional to the pair contribution to the Green-Kubo integrand for the bulk viscosity.

\section{DIFFUSION APPROXIMATION}

In the correlation function $\rho_{l}$, only the relative coordinate of the tagged particles 1 and 2 enters. For very long times these particles move independently most of the time, and each particle will suffer many collisions. In good approximation we can therefore assume that for both particles the space-time dependence is described by the self-diffusion equation. The time scale at which molecular scale effects are important may be estimated to be of the order of the time a particle needs to diffuse over a hard sphere diameter. With the Einstein relation $\left\langle(\Delta R(t))^{2}\right\rangle=2 D t$ we find $t_{\sigma}=\sigma^{2} / 2 D$, where $D$ is the self-diffusion constant. Using the Enskog value for the self-diffusion constant, ${ }^{(7)}$ we find at the density $n \sigma^{3}=0.884$ that $t_{\sigma}=314 t_{e}$. Already this simple argument shows that molecular scale effects can be important even for very large times.

In order to describe $\rho_{t}$, we use the diffusion equation for the relative 
coordinate of particles 1 and 2, also called the relative particle. Such an approach is very similar to making a mode coupling assumption, using ordinary diffusion modes. These two approaches can be related to one another through a spatial Fourier transform. However, the use of a diffusion equation suggests a number of improvements over the simplest approximation.

For the relative particle the diffusion equation reads

$$
\partial_{t} \hat{f}(\mathbf{r}, t)=2 D \Delta \hat{f}(\mathbf{r}, t)
$$

This can be related to the correlation function $\rho_{l}(t)$ by imposing the initial condition

$$
\hat{f}(\mathbf{r}, t=0)=\sigma Y_{l m}(\hat{\mathbf{r}}) \delta(r-\sigma)
$$

Due to rotational invariance around $\mathbf{r}=0$ we then have at all times

$$
\hat{f}(\mathbf{r}, t)=Y_{l m}(\hat{\mathbf{r}}) f(r, t)
$$

The function $\rho_{l}(t)$ is obtained from $f$ through

$$
\rho_{l}(t)=n \sigma^{3} g(\sigma) \cdot f(\sigma, t)
$$

In the simplest approximation we assume that the diffusion takes place in a homogeneous medium. Furthermore, we impose the boundary conditions that $f(r, t)$ is well behaved at $r=0$ and $r=\infty$. This diffusion problem is a standard problem in mathematical physics and can be solved by means of separation of variables. ${ }^{(8)}$ For $\rho_{l}$ we find

$$
\rho_{l}^{(0)}(t)=n \sigma^{3} g(\sigma)\left(\frac{\sigma^{2}}{4 D t}\right) \exp \left(-\frac{\sigma^{2}}{4 D t}\right) I_{l+1 / 2}\left(-\frac{\sigma^{2}}{4 D t}\right)
$$

with $I_{l+1 / 2}$ a modified Bessel function. Mode coupling theory produces the same results, multiplied by an extra factor of $g(\sigma) \cdot{ }^{(6)}$ For $l=0$ the solution (2.5) can also be written as

$$
\rho_{0}^{(0)}(t)=n \sigma^{3} g(\sigma) \frac{1-\exp \left(-\sigma^{2} / 2 D t\right)}{\left(8 \pi D t / \sigma^{2}\right)^{1 / 2}}
$$

This approximation, however, is somewhat crude, as recollisions are ignored entirely. One of the effects of recollisions between the two tagged particles is to ensure that they never overlap. So all recollisions can be taken into account at once by imposing that the probability flux through a spherical shell with radius $\sigma$ is zero,

$$
\left.\frac{\partial}{\partial r} f(r, t)\right|_{r=\sigma}=0
$$


The resulting diffusion problem again can be solved by means of separation of variables. ${ }^{(8)}$ For $\rho_{l}$ we find

$$
\rho_{l}^{(1)}(t)=\frac{2 n \sigma^{2} g(\sigma)}{\pi} \int_{0}^{\infty} d k \frac{\exp \left(-4 k^{2} D t\right)}{k^{2}\left\{\left[n_{l}^{\prime}(k \sigma)\right]^{2}+\left[j_{l}^{\prime}(k \sigma)\right]^{2}\right\}}
$$

where $j$ and $n$ are spherical Bessel functions, and a prime denotes differentiation with respect to argument. For $l=0$ this integral can be performed and we have

$$
\rho_{0}^{(1)}(t)=\frac{1}{\left(2 \pi D t / \sigma^{2}\right)^{1 / 2}}-e^{2 D t / \sigma^{2}} \operatorname{erfc}\left(\frac{2 D t}{\sigma^{2}}\right)^{1 / 2}
$$

with erfe the complement of the error function.

From the solutions (2.5) and (2.8) the long-time behavior follows. We find that in the long-time limit inclusion of the recollisions gives a numerically different result, as we have

$$
\frac{\rho_{l}^{(1)}(t)}{\rho_{l}^{(0)}(t)} \rightarrow\left(\frac{2 l+1}{l+1}\right)^{2} \quad(t \text { large })
$$

Although we have summed over all recollisions, this model is not quite satisfactory. For very large times, when the mean square displacement is much larger than the hard sphere diameter, we expect that the probability density for finding the two tagged particles at a separation $r$ will be proportional to the equilibrium probability, at least for $r$ not too large. So we expect that for large $t$

$$
\left\langle\delta\left(r_{12}(0)-\sigma\right) \delta\left(r_{12}(t)-r\right)\right\rangle=C(t) g_{2}(r)
$$

This asymptotic value is attained by the solution of a diffusion equation in a potential of mean force,

$$
V(r)=-\frac{1}{\beta} \log g_{2}(r)
$$

The diffusion equation now becomes, instead of (2.1),

$$
\partial_{t} \hat{f}(\mathbf{r}, t)=2 \nabla \cdot D\{\boldsymbol{\nabla}+\beta[\nabla V(r)]\} \hat{f}(\mathbf{r}, t)
$$

with the boundary condition

$$
\left.\left\{\frac{\partial}{\partial r}+\beta\left[\frac{\partial}{\partial r} V(r)\right]\right\} \hat{f}(\mathbf{r}, t)\right|_{r=\sigma}=0
$$


To be fair, standard mode coupling theory partly takes into account the potential of mean force through the extra factor of $g(\sigma)$ mentioned below (2.5). However, the potential of mean force will also influence the intermediate-time dynamics.

The microscopic mechanism that gives rise to the potential of mean force is twofold. First, as already mentioned, the two particles collide with each other. This is taken into account by the boundary condition (2.14). Another mechanism is that if the two particles are close, they will inhibit certain collisions by third particles. This inhibition effect we have taken into account through the potential of mean force. Again, this is an approximation, as the presence of a particle close by may subtly alter the dynamics as the distribution of collisions gets changed. It has been suggested that the effect of this is that the diffusion constant is increased, and becomes position and direction dependent (see, for instance, ref. 9). Here we have taken the simplest approach and used a constant selfdiffusion coefficient, and also ignored a possible time dependence. This can only be justified a posteriori through a comparison with simulation data.

The diffusion equation (2.13) was solved numerically. This was done by representing the function $f(r, t)$ on a finite number of positions (we used $300)$. The differential operators occurring in (2.13) are then replaced by difference operators and the diffusion equation takes on the form

$$
\frac{\partial}{\partial t} f\left(r_{i}, t\right)=\sum_{j} M_{i j} f\left(r_{j}, t\right)
$$

As the matrix $M$ is tridiagonal, its eigenvalues can be computed efficiently. The boundary condition at infinity is taken care of by imposing $f(R, t)=0$ for some large $R$; we took $R=10 \sigma$. For the pair correlation function we used the Percus-Yevick expression, including the Verlet-Weiss correction (see, e.g., ref. 10). In Fig. 1 we compare the solutions to the three different approximations for $\rho_{2}(t)$. We notice that the various approximations lead to rather different results. When comparing $\rho^{(1)}$ to $\rho^{(0)}$, we notice that including the recollisions has a marked effect, not only for short and intermediate times, but also for the prefactor of the long-time tail. For short times the diffusion is effectively one dimensional, as the particles do not diffuse over a distance large enough for the shape of the sphere to become significant. However, for short times the solution in the potential of mean force at $n \sigma^{3}=0.884$ is found to satisfy a $t^{-1 / 2}$ behavior only for times shorter than $2 D t / \sigma^{2} \equiv t^{*}<10^{-3}$. Also we have found that the $l$ dependence becomes important at times larger than $t^{*} \simeq 0.1$.

In Fig. 2 we compare our solution to the diffusion equation with a potential of mean force to the MD data of Ladd et al. ${ }^{(1,2)}$ We have used 


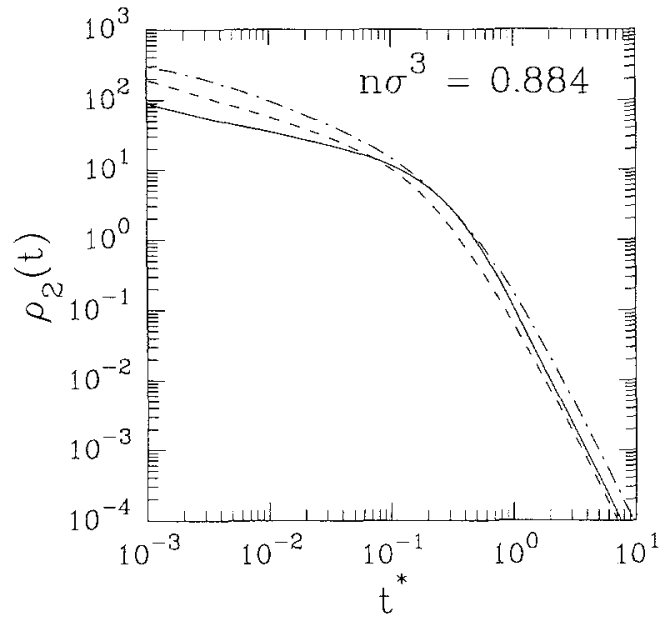

Fig. 1. Three solutions to the diffusion equation for $\rho_{2}(t)$ at high density. The time is in diffusion time units, $t^{*}=2 D t / \sigma^{2}$. Solid line: $\rho^{(\phi)}(t)$, effective potential approach. Dot-dashed line: $g(\sigma) \rho^{(1)}(t)$. In this approximation all recollisions are summed. Dashed line: $g(\sigma) \rho^{(0)}(t)$, simplest theory.

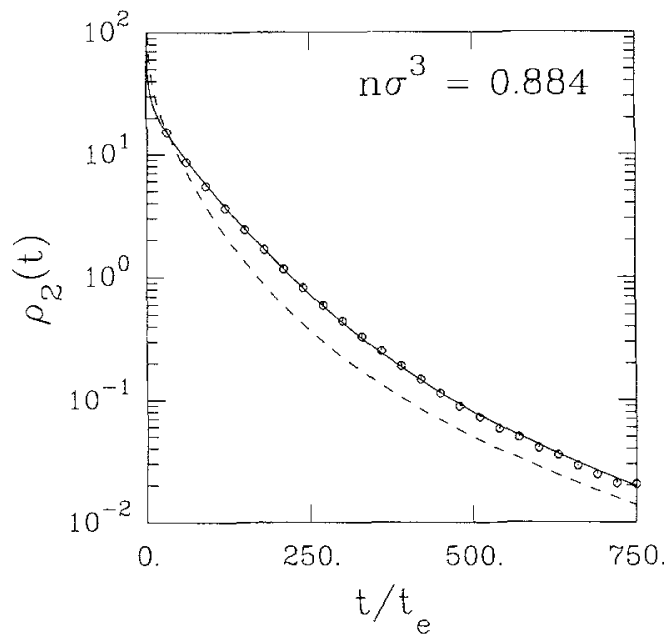

Fig. 2. Comparison of the simulation results of Ladd et al. ${ }^{(2)}$ for $\rho^{(K)}(t)$ (circles) and the solution of the diffusion equation in a potential of mean force (solid line). The error in the simulation data is about $2 \times 10^{-3}$. We have used $D=0.695 D_{e}$ so as to obtain the best fit for $t<450 t_{e}$. With this $D$ we have $t / t_{e}=450 t^{*}$. For comparison we also plot $g(\sigma) \rho^{(0)}(t)$ (dashed line), using the same $D$, as an even smaller $D$ would be needed to improve the fit. 
$D=0.695 D_{e}$, which is significantly lower than the molecular dynamics values $D=0.76 D_{e}$ reported by Alder et al. ${ }^{(11)}$ and $D=0.78 D_{e}$ found by Erpenbeck and Wood. ${ }^{(12)}$ The agreement is excellent, the maximum difference being about $5 \%$. The data of Ladd et al. span a time range from $t=30 t_{e}$ to $t=750 t_{e}$. Notice that such a close agreement requires a selfdiffusion constant accurate to within about $2 \%$, as in the diffusion approximation the correlation function $\rho_{2}(t)$ is proportional to $(D t)^{-7 / 2}$ for very long times. Similar results are found at $n \sigma^{3}=0.943$, but at $n \sigma^{3}=0.786$ somewhat larger differences appear at long times.

\section{DISCUSSION}

The result presented in Fig. 2 demonstrates that already a relatively simple theory is capable of describing the orientational pair correlation functions. The main input quantity is the diffusion constant used. We have shown that the simulation data can be very accurately reproduced with a diffusion constant that is somewhat lower than the self-diffusion constant found in MD simulations. This is an indication that the self-diffusion constant depends on the relative position of the two tagged particles.

Our calculations show that the stretched exponential behavior found by Ladd et al. can be understood as a transition phenomenon from the intermediate-time $t^{-1 / 2}$ behavior to the asymptotic $t^{-7 / 2}$ tail. It would be very interesting to make a similar comparison with simulations for the other angular correlation functions, in particular for $\rho_{0}(t)$.

The velocity factorization found by Ladd $e t$ al. is in a sense confirmed here, as the diffusion equation models $\rho^{(L)}(t)$, whereas the simulations are for $\rho^{(K)}(t)$.

The arguments used are of a hydrodynamic nature. So the theory presented should be applicable for other simple fluids as well. Natural but very difficult extensions of the theory presented here go in two directions. First there is the transition from the short-time behavior to the intermediate- to long-time behavior discussed here. It seems likely that an accurate sequence of descriptions will go through the following stages: At very short time the pseudo-Liouville operator has to be used, for intermediate times $\left(1<t / t_{e}<10\right)$ the Liouville operator can be approximated by an appropriate sum of Enskog operators, and for longer times this sum of Enskog operators can be replaced by the diffusion operator. We are presently investigating along these lines.

Another natural extension is to attempt to give an accurate description of the full stress tensor correlation function and related ones. The existing calculations ${ }^{(4,5)}$ generally show the trend of predicting a too rapid decay 
of the stress tensor autocorrelation function at times of the order of $15 t_{e^{*}}{ }^{(13)}$ In view of the results presented in Fig. 1, an extension of the ideas presented here is likely to improve on this.

\section{ACKNOWLEDGMENTS}

We thank T. R. Kirkpatrick for drawing our attention to this problem and for valuable remarks on the manuscript. We also wish to thank Tony Ladd for sharing his simulation results with us prior to publication.

\section{REFERENCES}

1. A. J. C. Ladd, W. E. Alley, and B. J. Alder, J. Stat. Phys. 48:1147 (1987).

2. A. J. C. Ladd and B. J. Alder, Preprint, UCRL; J. Stat. Phys., submitted; private communication.

3. H. van Beijeren, Phys. Lett. 105A:191 (1984).

4. T. R. Kirkpatrick and J. C. Nieuwoudt, Phys. Rev. A 33:2651 (1986).

5. I. M. de Schepper, A. F. E. M. Haffmans, and H. van Beijeren, Phys. Rev. Lett. 57:1715 (1986).

6. T. R. Kirkpatrick, private communication, cited in ref. 2.

7. P. Résibois and M. de Leener, Classical Kinetic Theory of Fluids (Wiley, New York, 1977).

8. I. N. Sneddon, Elements of Partial Differential Equations (McGraw-Hill, New York, 1957); B. M. Budak, A. A. Samarskii, and A. N. Tikhonov, A Collection of Problems on Mathematical Physics (Pergamon, New York, 1964).

9. G. T. Evans and B. Kumar, J. Chem. Phys. 90:1804 (1989).

10. J. P. Hansen and I. R. McDonald, Theory of Simple Liquids (Academic, London, 1976).

11. B. J. Alder, D. M. Gass, and T. E. Wainwright, J. Chem. Phys. 53:3813 (1970).

12. J. J. Erpenbeck and W. W. Wood, Phys. Rev. A 32:412 (1985).

13. J. J. Erpenbeck, Phys. Rev. A 38:6255 (1988). 